\title{
Sequences of Abstract Nonbiological Stimuli Share Ventral Premotor Cortex with Action Observation and Imagery
}

\author{
Ricarda I. Schubotz and D. Yves von Cramon \\ Department of Neurology, Max-Planck-Institute for Human Cognitive and Brain Sciences, 04103 Leipzig, Germany
}

Activation triggered by either observed or imagined actions suggests that the ventral premotor cortex (PMv) provides an action vocabulary that allows us to detect and anticipate basically invariant perceptual states in observed actions. In the present study, we tested the hypothesis that the same PMv region is also recruited by nonbiological (abstract) stimulus sequences as long as the temporal order of stimuli has to be processed. Using functional magnetic resonance imaging, we instructed participants to assess expected outcomes in observed actions [external biological cues (EB)], motor imagery [internal biological cues (IB)], or geometrical figure sequences [external nonbiological cues (EN)]. As hypothesized, we found that each condition elicited significant activation within PMv [left hemisphere, Brodman Area (BA) 6], in contrast to a sequential target detection control task. In addition, cue-specific activations were identified in areas that were only engaged for biologically (action) cued (EB, IB) and nonbiologically cued (EN) tasks. Biologically cued tasks elicited activations within inferior frontal gyri adjacent to PMv (BA 44/45), in the frontomedian wall, the extrastriate body area, posterior superior temporal sulci, somatosensory cortices, and the amygdala-hippocampal-area, whereas the nonbiologically cued task engaged presupplementary motor area, middle frontal gyri, intraparietal sulci, and caudate nuclei of the basal ganglia. In sum, findings point to a basic premotor contribution to the representation or processing of sequentially structured events, supplemented by different sets of areas in the context of either biological or nonbiological cues.

Key words: premotor cortex; temporal order; perceptual prediction; action observation; biological motion; motor imagery; fMRI; sequencing; prospective attention

\section{Introduction}

The ability to anticipate a perceptual event on the basis of internal or external cues speeds up our receptive processes and enables us to react quickly and appropriately. It plays a fundamental role in incredibly different domains such as motor control, during which "internal forward models" turn motor commands into expected sensory consequences (Jordan, 1996), and also in social life and communication, during which saying "I know what he is doing" expresses a conscious awareness of an agent's future goal (Gallese and Goldman, 1998); however, the neural constituents contributing to anticipatory processes are still obscure.

Conceptually, the formation of expectations has to rely on the representation of sequentially structured events, something like "A precedes B." One of the open questions that has not yet been tested directly is whether sequential representations based on biological cues (observed or imagined actions) and those based on nonbiological cues (abstract, sequentially ordered stimuli) recruit overlapping brain networks, i.e., whether cue-independent areas are engaged in sequential representation.

Received Feb. 11, 2004; revised May 3, 2004; accepted May 3, 2004.

We thank Sophie Manthey for the stimulus material, Andrea Gast-Sandmann for the figures, Ann-Shirley Rueschemeyer for proof reading and suggestions, Uta Wolfensteller, Thomas Jacobsen, Evelyn Ferstl, Marcel Brass, and Christian Fiebach for helpful comments on this manuscript, Gaby Lohmann and Karsten Müller for support in MRI statistics, and Katrin Sakreida for experimental assistance.

Correspondence should be addressed to Ricarda I. Schubotz, Department of Neurology, Max-Planck-Institute for Human Cognitive and Brain Sciences, P.0. Box 500 355, 04103 Leipzig, Germany. E-mail schubotz@cbs.mpg.de.

DOI:10.1523/JNEUROSCI.1169-04.2004

Copyright $\odot 2004$ Society for Neuroscience $\quad$ 0270-6474/04/245467-08\$15.00/0
A candidate area is the ventral premotor cortex (PMv), which is engaged by covert stages of action (Decety et al., 1994; Jeannerod and Decety, 1995; Hanakawa et al., 2003) and also by sequential tasks that operate on various nonbiological cues [for meta-analysis and overview, see Schubotz and von Cramon (2003)]. Animal data suggest that PMv houses an action repertoire with key constituents that are sensorimotor neurons, tuned to the accomplishment of different action goals such as grasping or reaching (Rizzolatti and Fadiga, 1998). Most interesting for the present purpose, however, is that many of those PMv neurons also show a temporal tuning, that is, they are specifically engaged in parts of an action such as hand aperture or hand closure during grasping (Rizzolatti et al., 1988). Temporal tuning, as a core element of sequential representations inherent to movement and action, manifests also in dorsal PM (Boussaoud et al., 1998) and medial PM [supplementary motor area (SMA)] (Shima and Tanji, 2000). Endowed with these particular neuronal properties and given its robust involvement in nonbiologically cued sequential representations, the question arises whether PMv may be exploited for both biological and nonbiological (abstract) sequential representations (Schubotz and von Cramon, 2004).

The present study investigated this hypothesis using functional magnetic resonance imagining (fMRI). First, we aimed to identify a PMv region that is activated both by observation of biological motion [external biological cues (EB)] and by imagined action [internal biological cues (IB)]. For both EB and IB, we used a predictive instruction that allowed us to ensure and test 


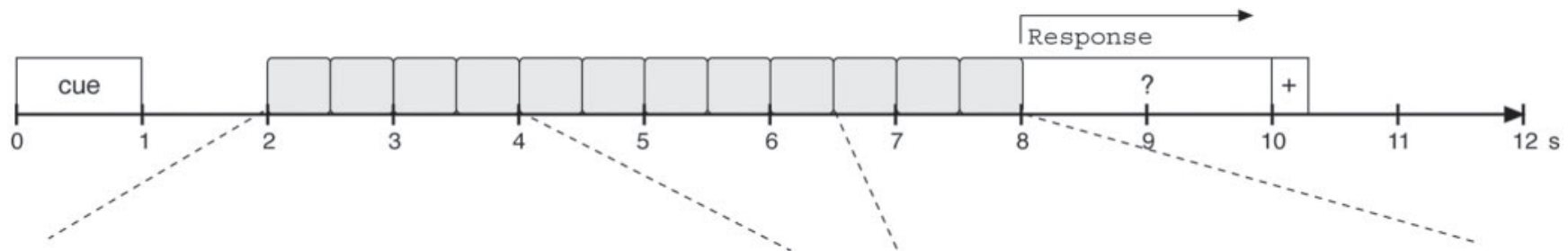

EB (external biological cues): Goal prediction in observed action

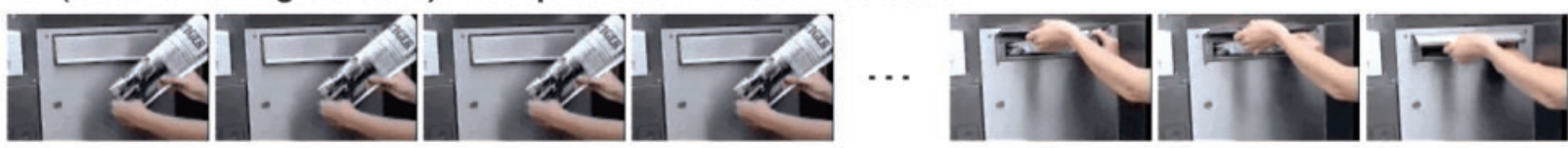

IB (internal biological cues): Effect prediction in motor imagery

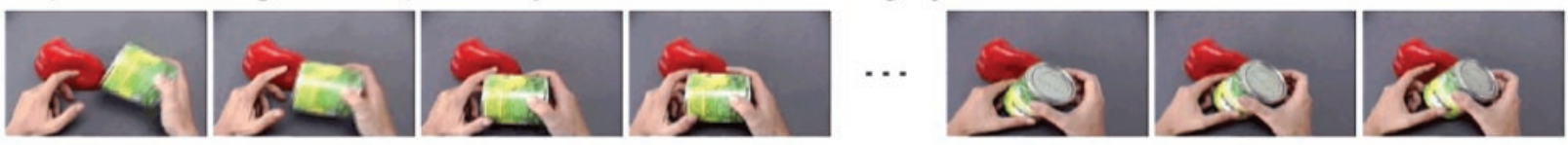

\section{EN (external non-biological cues): End prediction in abstract sequences}

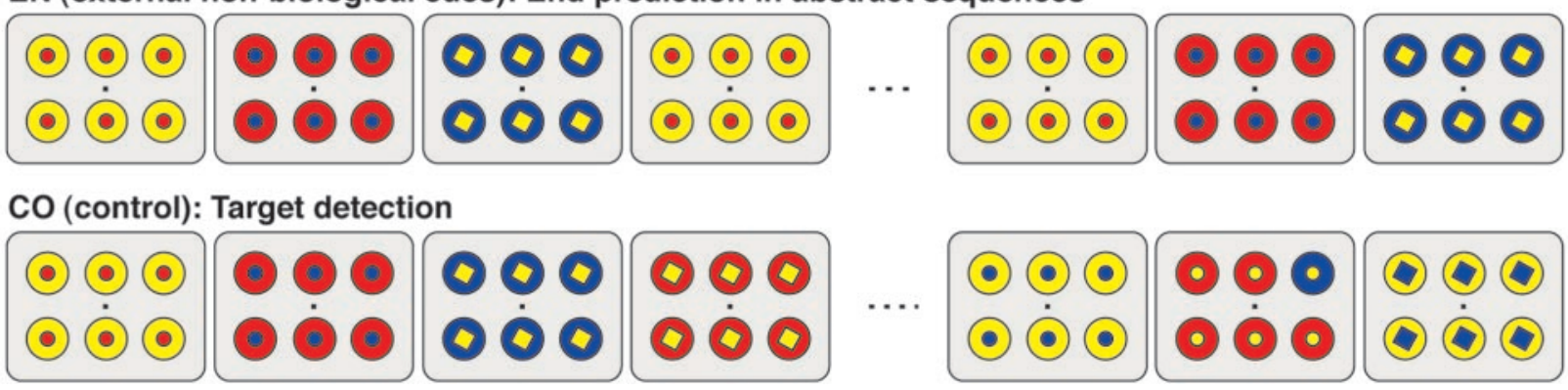

Figure 1. Experimental conditions. All trials followed the same temporal schema, depicted in the top panel. Examples for each condition (EB, IB, EN) are depicted in the bottom panels. In action observation (EB), participants were asked to indicate whether the presented action was performed correctly; in motor imagery (IB), they were asked whether the object held and turned within the actor's hands could be manually compressed; and in non-biological sequences (EN), they were asked whether a sequence of abstract figures followed a regular order or if the order was violated within the last three stimuli of a trial. In the control condition $(C O)$, participants were requested to indicate whether one frame contained a circle figure that differed from the concurrently presented ones. Correct responses for the examples given in this figure were yes (observed action was performed correctly) (EB), no (manipulated object is not soft) (IB), yes (order of pictures was correctly repeated) (EN), and yes (a deviant element was detected in one of the last pictures) (CO).

on-line whether participants' attention was focused on the goal of the currently observed or imagined action. If one assumes an area within the PMv that contributes to representation of goaldirected action, this area should be identified by this procedure. Second, we tested whether the same PMv area is also engaged in predictions that are cued by abstract nonbiological motion [external nonbiological cues (EN)], as implemented in a serial prediction task (SPT) (Schubotz, 1999; Schubotz and von Cramon, 2003). Although biological and nonbiological cues were expected to recruit additionally different areas, the representation of a minimal sequence of invariant perceptual states was intended to be shared by all experimental conditions. This hypothesis followed the rationale of Byrne and Russon (1998), according to which essential goal and subgoals of an action recur in every effective action sequence, whereas the irrelevant details of precisely how each of these intermediate states is achieved will vary between occasions without affecting efficiency. As such, goals and subgoals are the predictable constituents of an observed action; they are the most invariant perceptual states that an agent plans to cause and that both the actor and a potential observer expect (and hence predict) to perceive.

\section{Materials and Methods}

Participants. Eighteen right-handed, healthy young volunteers (10 female, 8 male; age range, 21-34 years; mean age 25.9 years) participated in the study. After being informed about potential risks and screened by a physician of the institution, subjects gave informed consent before par- ticipating. The experimental standards were approved by the local ethics committee of the University of Leipzig. Data were handled anonymously.

Stimuli. The paradigm comprised three experimental conditions (Fig. 1) and one baseline condition. Thirty-six trials were presented per condition, and conditions were presented in random order (mixed-trial design). Within each trial of each condition, visual stimulation lasted $6 \mathrm{sec}$ and was preceded by a $2 \mathrm{sec}$ verbal task cue; stimulation was followed by the response phase and an intertrial interval that lasted $4 \mathrm{sec}$ in total.

In condition $\mathrm{EB}$, movies showing bimanual actions were presented that were familiar from everyday life; for example, cutting a piece of paper with scissors, lacing a shoe, shuffling cards, taking butter with a knife, or opening a can. Movies always showed both hands and two objects. In $50 \%$ of EB trials, the presented action resulted in an action slip that rendered the final achievement of the intended action goal unlikely. As confirmed by the participants' reports after the experiment, these action slips were not detected immediately at the beginning of the movie but required a careful observation until the end of the presentation. Conversely, because participants were aware of action slips, they reported that they observed carefully all EB trials, including those presenting correct actions, until the movie ended to make an appropriate choice.

In condition IB, movies were presented showing two hands holding and moving one of two concurrently presented objects (the second, irrelevant object was presented to balance the number of objects presented in $\mathrm{EB}$ and IB). In contrast to condition EB, however, the displayed hands did not perform a goal-directed action but just "played around" with the object. This motion was not task relevant in IB (see Task instructions below) but was used only to match and balance the perceptual motion information provided by each of the experimental conditions. The stim- 
ulus material used in conditions EB and IB was also partly used in a previous fMRI study (Manthey et al., 2003).

In condition EN, 12 different objects were used, each composed of a 25 mm circle ( 0.148 of visual angle) and a slightly smaller geometrical form placed in its center. In six objects, this was a $14 \mathrm{~mm}$ square, and in the six other objects it was a $10 \mathrm{~mm}$ circle. The big circle and the small form were colored red, yellow, or blue, in such a manner that objects were always two-colored. The same stimulus material was used in a previous study (Schubotz and von Cramon, 2001). On each screen, an array of six identical objects was presented. The size of the array was matched with that in conditions $\mathrm{EB}$ and $\mathrm{IB}\left(<5^{\circ}\right.$ of visual angle). Within one EN trial, a sequence of 12 screens was presented subsequently for $500 \mathrm{msec}$ each, with objects changing from one screen to the next. The succession of the objects presented within each trial exposed a regular order such that three objects built up a sequence that was repeated four times. In $50 \%$ of the EN trials, the order of the last two screens was flipped, resulting in a violation of the sequential object order. In the control condition (CO), the same type of stimulus was presented as in the EN condition, but the screen-to-screen order of objects was completely irregular (random). In $50 \%$ of the CO trials, one of the last two screens was a predefined target, containing one object of deviant color-form combination, as compared with the other five concurrently presented stimuli.

Task instructions. For conditions EB and EN, participants were instructed to attend to the stimulus presentation and to predict how this stimulation would evolve. In the EB condition, this instruction directed the participants' attention to the intended action goal, whereas in the EN condition, participants' attention was drawn to the sequential object order. In both EB and EN, prediction was tested by a forced-choice response after the end of the stimulus presentation. After action observation in $\mathrm{EB}$, participants had to indicate whether the action was performed in a goal-directed manner (button "yes") or not (button "no"). After abstract motion observation in EN, participants had to indicate whether the object sequence was regular until the end of presentation (button yes) or not (button no).

In condition IB, participants were asked to identify the displayed object and judge whether it was a soft object, i.e., whether it would be deformed when compressed with the hand (button yes) or not (button no). By this instruction, participants were required to imagine themselves compressing the observed object and to make a judgment on the basis of the imagined tactile-kinesthetic outcome of this action. It is important to note that the turning of the object in the actor's hands provided no relevant information for the task itself, i.e., objects were not compressed by the displayed hands, but was rather used to adapt the amount of motion information provided by IB to that provided in EB and EN.

After trials of condition CO, participants had to indicate whether the trial contained the predefined target array (button yes) or not (button no). Because the sequential order of stimuli was irrelevant for identifying targets in the baseline condition, this condition did not require prediction.

Data acquisition. Participants were instructed before the MRI experiment. In the MRI session, subjects were supine on the scanner bed with their right index and middle finger positioned on the response buttons. To prevent postural adjustments, the subjects' arms and hands were carefully stabilized by tape. In addition, form-fitting cushions were used to prevent arm, hand, and head motion. Participants were provided with earplugs to attenuate scanner noise.

Imaging was performed at 3T on a Bruker Medspec 30/100 system equipped with the standard birdcage head coil. Sixteen axial slices (field of view $192 \mathrm{~mm}$; $64 \times 64$ pixel matrix; thickness $5 \mathrm{~mm}$; spacing $2 \mathrm{~mm}$ ) parallel to bicommissural line (AC-PC) were acquired using a singleshot gradient echo-planar imaging (EPI) sequence (echo time, $30 \mathrm{msec}$; flip angle, $90^{\circ}$; repetition time, $2000 \mathrm{msec}$ ) sensitive to blood oxygenation level-dependent contrast. A set of two-dimensional (2D) anatomical images was acquired for each subject immediately before the functional experiment, using a modified driven equilibrium Fourier transformation $(\mathrm{MDEFT})$ sequence $(256 \times 256$ pixel matrix $)$. In a separate session, high-resolution whole-brain images were acquired from each subject to improve the localization of activation foci using a T1-weighted three- dimensional (3D) segmented MDEFT sequence covering the whole brain.

Data analysis. The MRI data were processed using the software package LIPSIA (Lohmann et al., 2001). Functional data were corrected for motion using a matching metric based on linear correlation. To correct for the temporal offset between the slices acquired in one scan, a sincinterpolation based on the Nyquist-Shannon theorem was applied. A temporal high-pass filter with a cutoff frequency of $1 / 96 \mathrm{~Hz}$ was used for baseline correction of the signal. No spatial filter was applied. To align the functional data slices with a 3D stereotactic coordinate reference system, a rigid linear registration with $6 \mathrm{df}$ ( 3 rotational, 3 translational) was performed. The rotational and translational parameters were acquired on the basis of the MDEFT and EPI-T1 slices to achieve an optimal match between these slices and the individual 3D reference data set. This 3D reference data set was acquired for each subject during a previous scanning session. The MDEFT volume data set with 160 slices and $1 \mathrm{~mm}$ slice thickness was standardized to the Talairach stereotactic space (Talairach and Tournoux, 1988). The rotational and translational parameters were subsequently transformed by linear scaling to a standard size. The resulting parameters were then used to transform the functional slices using trilinear interpolation, so that the resulting functional slices were aligned with the stereotactic coordinate system. This linear normalization process was improved by a subsequent processing step that performed an additional nonlinear normalization (Thirion, 1998). Slice gaps were interpolated to generate output data with a spatial resolution of $3 \times 3 \times 3 \mathrm{~mm}$.

The statistical evaluation was based on a least-squares estimation using the general linear model for serially autocorrelated observations (Friston, 1994; Friston et al., 1995a,b; Worsley and Friston, 1995). The design matrix was generated with a synthetic hemodynamic response function (Josephs et al., 1997; Friston et al., 1998) and its first and second derivative. To tap prediction-related processes, brain activations were analyzed in an event-related design, time-locked to a point $2 \mathrm{sec}$ after stimulus onset. Only correctly answered trials entered the analysis. The model equation, including the observation data, the design matrix, and the error term, was convolved with a Gaussian kernel of dispersion of $4 \mathrm{sec}$ full width at half maximum to deal with the temporal autocorrelation (Worsley and Friston, 1995). In the following, contrast images, i.e., estimates of the raw-score differences between specified conditions, were generated for each participant. The single-participant contrast images were then entered into a second-level random effects analysis for each of the contrasts. The group analysis consisted of a one-sample $t$ test across the contrast images of all participants that indicated whether observed differences between conditions were significantly distinct from zero (Holmes and Friston, 1998). Subsequently, $t$ values were transformed into $z$ scores. To protect against false positive activations, only regions with $z$ score $>3.09$ ( $p<0.001$; uncorrected $)$ and with a volume $>540$ $\mathrm{mm}$ (20 measured voxels) were considered. All reported activations survived a threshold corresponding to $p<0.05$ (corrected for multiple comparisons) at the cluster level.

\section{Results}

\section{Behavioral performance}

Performance was assessed by error rates. A repeated-measures ANOVA with the two-level factor TASK (prediction, control) indicated a significant main effect $\left(F_{(1,17)}=121.9 ; p<0.0001\right)$, with an error rate of $14.1 \%$ for prediction and $1.0 \%$ for condition CO. A repeated-measures ANOVA with the three-level factor TYPE $(\mathrm{EB}, \mathrm{EN}, \mathrm{IB})$ also indicated a significant main effect $\left(F_{(2,34)}=7.9\right.$; $p<0.006$ ), with an error rate of $17.9 \%$ for $\mathrm{EB}, 14.8 \%$ for $\mathrm{EN}$, and $9.7 \%$ for IB.

\section{MRI data}

Contrasted with $\mathrm{CO}$, each condition EB, IB, and EN caused significant and overlapping activation within the left PMv (Fig. 2). The foci of these activations were almost identical for the prediction conditions, located at $x=-50, y=7, z=29$ for EB, at $x=$ 

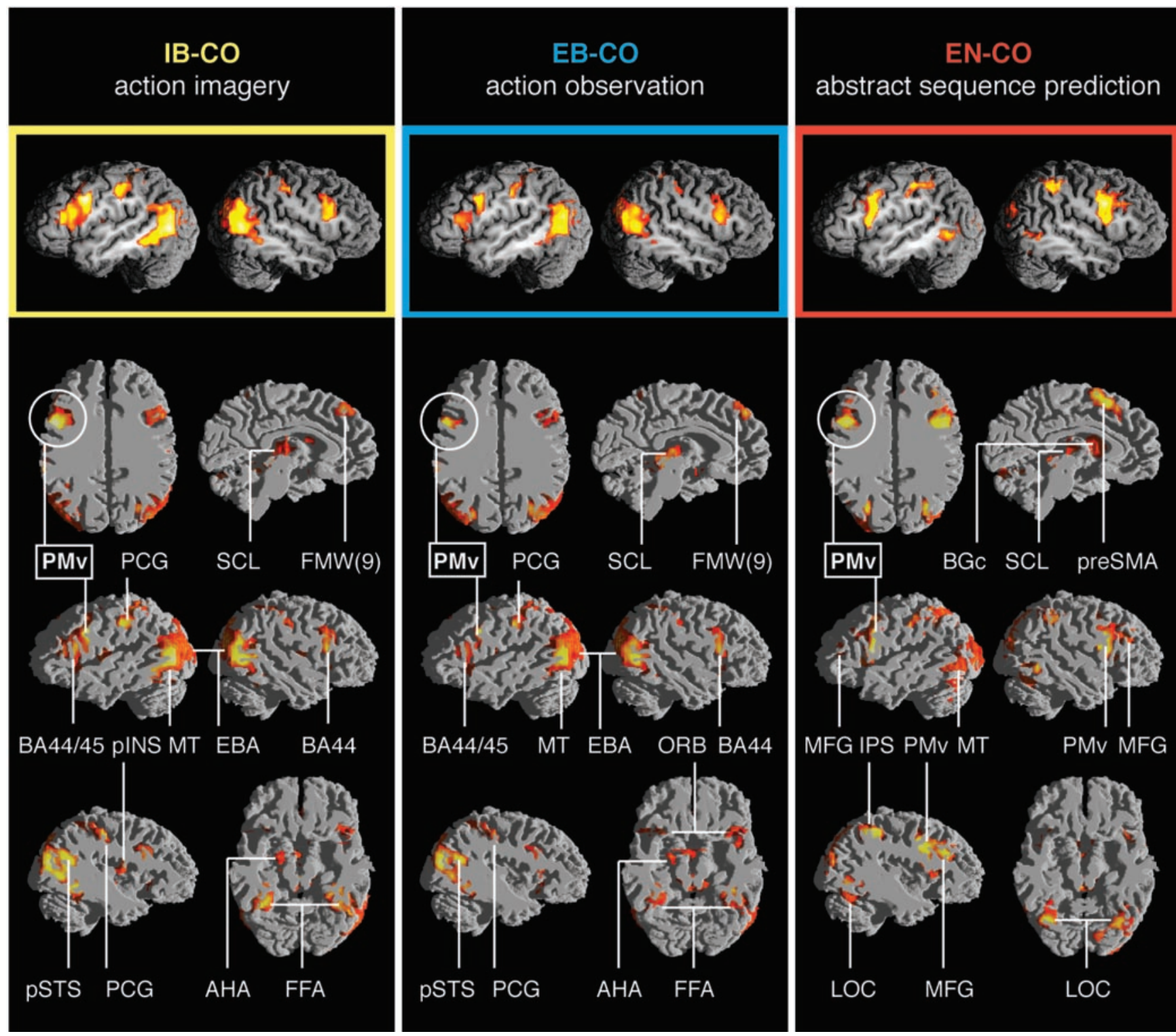

Figure 2. Brain correlates of experimental tasks. Group-averaged ( $n=18)$ statistical maps of significantly activated areas for prediction based on IB, EB, and EN cues, as opposed to nonpredictive control condition (CO).Z-maps were thresholded at $z=3.09$ ( $p<0.05$ corrected). Top panel shows left (left) and right (right) sagittal sections through activations within ventral premotor cortex and adjacent $\mathrm{BA} 44 / 45$. In bottom panels, activation is overlaid onto a white matter segmentation as an anatomical reference image. For each vertical $\mathrm{Column}$ (IB-CO, EB-CO, and EN-CO), displayed slices cut through Talairach coordinates $z=29$ and $x=-3$ (top panel), $x=-48$ and $x=48$ (middle panel), and $x=42$ and $z=-6$ (bottom panel). Common activations within left PMv are highlighted. For anatomical abbreviations see Table 1 (available at www.jneurosci.org).

$-47, y=4, z=32$ for IB, and at $x=-44, y=7, z=32$ for EN (Table 1, available at www.jneurosci.org). Although right PMv activations also overlapped between all three conditions, a local activation maximum within the right PMv was found only for condition EN ( $x=45, y=7, z=26)$, whereas in conditions EB and $\mathrm{IB}$, right $\mathrm{PMv}$ activation rather extended from a more anterior activation focus in adjacent areas BA 44/45. Condition EN showed additional left PMv activation located inferiorly to the common peak $(x=-53, y=7, z=11)$. Moreover, each prediction contrast activated both superior colliculi and a left temporal region, corresponding to the motion area (MT) described in previous studies (Tootell et al., 1995).

Conditions using biological cues (EB and IB) shared a set of activations that were not significantly activated for nonbiological cues (EN). These included bilateral inferior frontal gyri within BA $44 / 45$, left frontomedian wall (FMW) within the vicinity of BA
$9 \mathrm{~m}$, bilateral extrastriate body area (EBA) in the temporooccipital cortex, a region within the posterior superior temporal sulci (pSTS) located $\sim 20 \mathrm{~mm}$ anterior to the EBA activation maximum, the collateral sulci within the anterior fusiform gyri corresponding to the fusiform face area (FFA), the amygdalahippocampal area (AHA) bilaterally, the left posterior insula (pINS), and both postcentral gyri corresponding to primarysecondary somatosensory cortex (SI, SII).

Finally, each condition also showed a set of regions to be specifically engaged, including the right posterior insula and the left cerebellar cortex close to the vermis, which were found only for contrast IB-CO, and bilateral foci within posterior lateral orbitofrontal cortex (BA 47), which were observed only for EB-CO. Finally, increased levels of activation for $\mathrm{EN}-\mathrm{CO}$ were found in the left presupplementary motor area (pre-SMA), the middle frontal gyri, the intraparietal sulci, bilateral heads of the caudate nuclei, and a ven- 


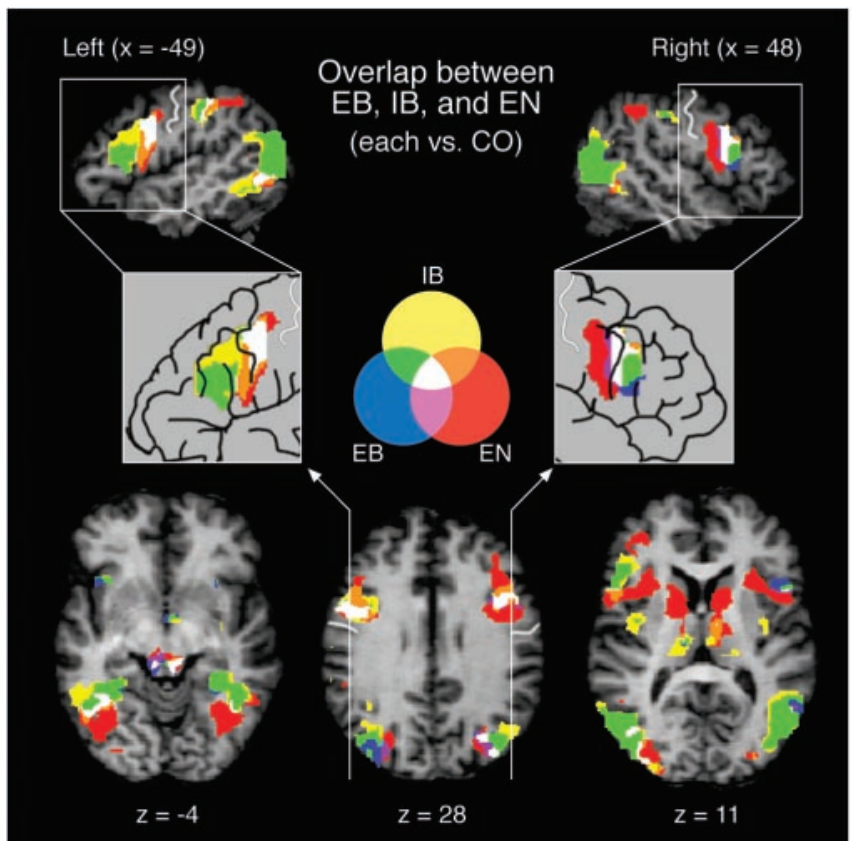

Figure 3. Overlap between experimental tasks. Significantly activated voxels of contrast IB-CO (yellow), EB-CO (blue), and EN-CO (red) are displayed as an overlay map. White fields show overlapping activations of all three conditions, whereas other colors indicate overlaps between two or three conditions (green indicates IB and EB, purple indicates EB and EN, and orange indicates EN and IB). The central sulcus is highlighted in white. The present study focused on the overlap of all conditions (white fields), which was especially prominent in the PMv. PMv activations are also indicated on brain schemas in the middle panel to focus on the anatomical localization (sulci were redrawn from the reference brain). Further overlap between all conditions was located in the left motion area and the superior colliculi. Overlap between EB and IB was found in the fusiform face area, extrastriate body area, and inferior frontal gyri.

tral-caudal portion of the fusiform gyri probably corresponding to the lateral occipital complex (LOC). Figure 3 shows overlapping and nonoverlapping activations of contrasts EB-CO, IB-CO, and EN-CO superimposed on an individual brain.

\section{Discussion}

The PMv is suggested to play a role in covert stages of action, including those triggered by action observation and action imagery, but also in making predictions cued by nonbiological stimuli. Pursuing the view that these different domains engage the PMv because the representation of sequential events is common to all of them, the present study tested whether a common PMv region is activated by outcome-related tasks on action observation, action imagery, or abstract sequences. Although correlates of biologically and nonbiologically cued conditions clearly differed within other brain areas, the overlapping activations within PMv substantiate its contribution to sequential representations fed by different cues.

Premotor overlap of EB and IB confirms a strong coupling between observed and imagined actions in this cortical area, as already suggested by corticospinal facilitation effects and eventrelated brain potentials (Schnitzler et al., 1997; Hari et al., 1998; Senkfor et al., 2002; Patuzzo et al., 2003; Clark et al., 2004). The role of the PMv in this coupling has been specified as a matching between stored and observed actions (Jeannerod, 2001), a specific application of the general matching functions in PMv. In animal literature, the basis of perception-action matching has been conceptualized as a "vocabulary of motor acts" stored within the PMv (Rizzolatti et al., 1988; Rizzolatti and Fadiga,
1998). These vocabularies may be addressed either internally, i.e., by action planning, or by appropriate external stimuli, even in the absence of motor intention (Murata et al., 1997). Initially neutral with respect to their behavioral role (Fadiga et al., 2000), premotor "vocabularies" can hence be exploited (1) for behavior and imitation (production), when actions are generated from this vocabulary (Rizzolatti and Gentilucci, 1988; Rizzolatti et al., 1988; Jeannerod et al., 1995), and (2) for recognition and understanding (reception), when perceived objects or observed actions are mapped onto it (Di Pellegrino et al., 1992; Rizzolatti et al., 1996). Action goals rather than action means appear to be the core of this tight perception-action linkage: the majority of F5 neurons discharge during the execution of goal-directed actions, but not during comparable finger or hand movements (Rizzolatti et al., 1988), and many F5 neurons code movements that share the same goal but are performed with different effectors (Fadiga et al., 2000). This goal preponderance can also be tapped in human imitation ("theory of goal-directed imitation") (Wohlschläger et al., 2003), and both imitation and other paradigms focusing participants' attention on action goals were found to activate human PMv (Decety et al., 1997; Grezes et al., 1999; Chaminade et al., 2002, Johnson-Frey et al., 2003).

The present findings suggest that this apparently very flexible "action vocabulary" in the PMv can also be exploited, at least in humans, for much more abstract processes than assumed previously. Although abstract 2D color-form combinations used in conditions EN (or more generally, in SPTs) do not have a oneto-one motor implication, as real objects or observed actions have, the PMv appears to be able to generate short-term templates of such stimulus sequences and therewith provide a basis for perceptual predictions. Particularly in view of perceptual responses in monkey PMv, the acquisition and storage of abstract perceptual templates in human PMv is conceivable in principle. We have investigated this hypothesis in a series of fMRI studies, particularly varying the perceptual information that has to be predicted on the basis of regularly repetitive sequences. Condition EN confirmed that object prediction tasks, as opposed to predictions tuned by spatial or rhythmic information, cause activation within the PMv posteriorly, adjacent to the inferior frontal sulcus (Schubotz and von Cramon, 2001, 2002a,b,c; Schubotz et al., 2003). On the basis of the imaging literature (Schubotz and von Cramon, 2003), this activation falls in a premotor area that has been related to manual control and object attention. Using observation of object manipulation in condition EB and imagery of object manipulation in condition IB of the present study, we expected that prediction on the basis of object-specific cues (color and form) provided in EN would overlap with both EB and IB effects in this part of the PMv because of a habitually consolidated hand-object correspondence and hence object-property preference in this area. The abstractness of representation implicated here corresponds very nicely to the view that perceived events and to-be-produced events (action goals) are represented by composite codes of their "distal" features (Hommel et al., 2002). Precursors of this theory implicate the (pre)motor cortices in providing this coding interface (Liberman et al., 1967; Rizzolatti et al., 1987).

Beyond shared operations mediated by the PMv, activation in other brain areas reflected specifically either biologically or nonbiologically cued conditions. Particularly of interest, EB and IB activated BA 44/45 (including Broca's area and its right homolog). On the basis of the monkey literature, this activation may reflect responses of the so-called "mirror neurons" (Gallese et al., 1996; Rizzolatti et al., 1996), which appear to be endowed with 
the properties that simulation theories require (Gallese and Goldman, 1998; Carr et al., 2003; Miall, 2003). Hence, the template matching mediated by PMv-BA6 might have been supplemented, only in the case of biological cues, by goal representations in anteriorly adjacent PMv-BA 44 (Umiltà et al., 2001). According to this view, condition EB required one specific instance of an action (as shown in the movie) to be matched with a standard template of that action (invariant features), probably stored in BA 44 (Rizzolatti et al., 2001). Likewise, imageries required in IB were conceivably generated from such a standard template; however, because Broca's area is also known to mediate linguistic representations, especially in tasks that require strategic matching (Bookheimer, 2002), an alternative interpretation could come from ontogenetic and phylogenetic accounts, stressing the general role of Broca's area in hierarchically organized sequential behavior (Greenfield, 1991). Similar to the role of preSMA in the organization of abstract sequences (Shima and Tanji, 2000), BA 44 may contribute to the generation of higher-level organization in actions, running on lower-level, serially organized representations within adjacent PMv-BA6.

The remaining discussion will focus on additional areas specifically engaged for EB and IB, because areas exclusively activated by EN are a direct replication of previous findings and have been discussed previously (Schubotz and von Cramon, 2003). Moreover, some of these areas cannot be discussed in detail: SI/ SII and pINS, which can be taken to reflect tactile-kinesthetic imagery (Carlsson et al., 2000; Bushara et al., 2001; Yoo et al., 2003), and middle cerebellar cortex activity in IB, which we take to confirm the cerebellar role in predicting the specific sensory consequences of movements (Blakemore et al., 2001).

The FMW (BA 9m) is reciprocally connected with lateral PM (Barbas et al., 1999). Imaging studies relate activation within BA $9 \mathrm{~m}$ to (1) the ability to distinguish between actions executed by oneself and actions executed by others (Brass et al., 2001; Ruby and Decety, 2001) and (2) self-referential representations (Ferstl and von Cramon, 2002; Zysset et al., 2002) that help to distinguish between our own and another person's perspective (Frith and Frith, 1999; Gallagher et al., 2002; Gallagher and Frith, 2003). This interpretation fits well with our findings, because EB required participants to take the perspective of the presented actor and subsequently to distinguish carefully between the actor's performance and what the participant himself or herself would do to achieve the inferred goal. Although condition IB did not require such a perspective, participants had to identify an aimlessly manipulated object. We suggest that seeing this movement interfered with the requirement to imagine oneself pressing the object to determine its softness, i.e., a highly similar movement (Brass et al., 2001).

Posterior STS and bilateral EBA were activated by EB and IB. Although both areas are known to respond to biological stimuli, only EBA is contingent on the shape of a body being explicitly represented (Downing et al., 2001; Grossman and Blake, 2002), whereas pSTS is rather driven by dynamics of biological stimuli such as actions and gestures. Its putative homolog in macaques (anterior superior temporal sulcus) is known to respond to the observation of biological actions, just as F5c (Rizzolatti et al., 2001), and contributes to the recognition and understanding of the actions of other (Emery and Perrett 1994; Jellema et al., 2002), an interpretation that is paralleled in the human literature (for review, see Allison et al., 2000). The present pSTS coordinates resemble those reported especially for perception of hand (as compared with body) motion (Bonda et al., 1996). Posterior STS activation in conditions EB and IB corresponded nicely to acti- vation within two additional areas that are known to be connected with pSTS (Barbas, 1988; Amaral et al., 1992), the posterior lateral orbitofrontal cortex and the AHA. On the basis of a review on orbitofrontal cortex and amygdala (Adolphs, 1999), we take their activation in IB and EB to reflect the mediation between perceptual representations of the sight of conspecifics and retrieval of knowledge triggered by such stimuli. Finally, EB and IB caused specific activation within the FFA (Kanwisher et al., 1997), which is known to respond not only to faces but also to highly familiar objects (Gauthier et al., 1999). In contrast, the caudalventral sector of the LOC activated by condition EN responds more generally to object shapes (Grill-Spector et al., 2001). In sum, occipital and temporal activations consistently reflected specific requirements in the context of the interpretation of biological as opposed to nonbiological cues.

\section{Conclusion}

Recent research has discarded the classical "motor planning" concept of premotor cortex in favor of a multipurpose actionperception matching interface. The present findings suggest that this interface can be exploited for the representation of sequentially structured events in a broader range of behaviors as assumed previously. Although each implementation asks for additional contributions from a set of specialized areas, tasks that rely on sequentially structured information may always converge on the PMv, be it in the context of planned, imagined, executed, or observed actions or in spatiotemporal trajectories or abstract sequences defined by numerical, figural, or acoustic stimuli. Even in the absence of biologically or pragmatically meaningful stimuli, instructed prediction suffices to activate the PMv; however, that is not to say that only prediction engages PMv. Future studies must try to settle which common operations provided by the PMv are shared in biologically and nonbiologically cued nonmotor tasks.

\section{References}

Adolphs R (1999) Social cognition and the human brain. Trends Cogn Sci 3:469-479.

Allison T, Puce A, McCarthy G (2000) Social perception from visual cues: role of the STS region. Trends Cogn Sci 4:267-278.

Amaral DG, Price JL, Pitkaenen A, Carmichael ST (1992) Anatomical organization of the primate amygdaloid complex. In: The amygdala: neurobiological aspects of emotion, memory, and mental dysfunction (Aggleton JP, ed), pp 1-66. New York: Wiley.

Barbas H (1988) Anatomic organization of basoventral and mediodorsal visual recipient prefrontal regions in the rhesus monkey. J Comp Neurol 276:313-342.

Barbas H, Ghashghaei H, Dombrowski SM, Rempel-Clower NL (1999) Medial prefrontal cortices are unified by common connections with superior temporal cortices and distinguished by input from memory-related areas in the rhesus monkey. J Comp Neurol 410:343-367.

Blakemore SJ, Frith CD, Wolpert DM (2001) The cerebellum is involved in predicting the sensory consequences of action. NeuroReport 12:1879-1884.

Bonda E, Petrides M, Ostry D, Evans A (1996) Specific involvement of human parietal systems and the amygdala in the perception of biological motion. J Neurosci 16:3737-3744.

Bookheimer S (2002) Functional MRI of language: new approaches to understanding the cortical organization of semantic processing. Annu Rev Neurosci 25:151-188.

Boussaoud D, Jouffrais C, Bremmer F (1998) Eye position effects on the neuronal activity of dorsal premotor cortex in the macaque monkey. J Neurophysiol 80:1132-1150.

Brass M, Zysset S, von Cramon DY (2001) The inhibition of imitative response tendencies. NeuroImage 14:1416-1423.

Bushara KO, Wheat JM, Khan A, Mock BJ, Turski PA, Sorenson J, Brooks BR 
(2001) Multiple tactile maps in the human cerebellum. NeuroReport 12:2483-2486.

Byrne RW, Russon AE (1998) Learning by imitation: a hierarchical approach. Behav Brain Sci 21:667-684.

Carlsson K, Petrovic P, Skare S, Petersson KM, Ingvar M (2000) Tickling expectations: neural processing in anticipation of a sensory stimulus. J Cognit Neurosci 12:691-703.

Carr L, Iacoboni M, Dubeau MC, Mazziotta JC, Lenzi GL (2003) Neural mechanisms of empathy in humans: a relay from neural systems for imitation to limbic areas. Proc Natl Acad Sci USA 100:5497-5502.

Chaminade T, Meltzoff AN, Decety J (2002) Does the end justify the means? A PET exploration of the mechanisms involved in human imitation. NeuroImage 15:318-328.

Clark S, Tremblay F, Ste-Marie D (2004) Differential modulation of corticospinal excitability during observation, mental imagery and imitation of hand actions. Neuropsychologia 42:105-112.

Decety J, Perani D, Jeannerod M, Bettinardi V, Tadary B, Woods R, Mazziotta JC, Fazio F (1994) Mapping motor representations with positron emission tomography. Nature 371:600-602.

Decety J, Grezes J, Costes N, Perani D, Jeannerod M, Procyk E, Grassi F, Fazio F (1997) Brain activity during observation of actions. Influence of action content and subject's strategy. Brain 120:1763-1777.

Di Pellegrino G, Fadiga L, Fogassi L, Gallese V, Rizzolatti G (1992) Understanding motor events: a neurophysiological study. Exp Brain Res 91:176-180.

Downing PE, Jiang Y, Shuman M, Kanwisher N (2001) A cortical area selective for visual processing of the human body. Science 293:2470-2473.

Emery NJ, Perrett DI (1994) Understanding the intentions of others from visual signals: neurophysiological evidence. Curr Psychol Cogn 13:683-694.

Fadiga L, Fogassi L, Gallese V, Rizzolatti G (2000) Visuomotor neurons: ambiguity of the discharge or "motor" perception? Int J Psychophysiol 35:165-177.

Ferstl EC, von Cramon DY (2002) What does the frontomedian cortex contribute to language processing: coherence or theory of mind? NeuroImage 17:1599-1612.

Friston KJ (1994) Statistical parametric mapping. In: Functional neuroimaging (Thatcher RW, Hallet M, Zeffiro T, John ER, Huerta M, eds), pp 79-93. San Diego: Academic.

Friston KJ, Holmes AP, Poline JB, Grasby BJ, Williams CR, Frackowiak RSJ, Turner R (1995a) Analysis of MRI time-series revisited. NeuroImage 2:45-53.

Friston KJ, Holmes AP, Worsley KJ, Poline JP, Frith CD, Frackowiak RSJ (1995b) Statistical parametric maps in functional imaging: a general linear approach. Hum Brain Mapp 2:189-210.

Friston KJ, Fletcher P, Josephs O, Holmes A, Rugg MD, Turner R (1998) Event-related fMRI: characterizing differential responses. NeuroImage $7: 30-40$.

Frith CD, Frith U (1999) Interacting minds-a biological basis. Science 286:1692-1695.

Gallagher HL, Frith CD (2003) Functional imaging of "theory of mind". Trends Cogn Sci 7:77-83.

Gallagher HL, Jack AI, Roepstorff A, Frith CD (2002) Imaging the intentional stance in a competitive game. NeuroImage 16:814-821.

Gallese V, Goldman A (1998) Mirror neurons and the simulation theory of mind-reading. Trends Cogn Sci 2:493-501.

Gallese V, Fadiga L, Fogassi L, Rizzolatti G (1996) Action recognition in the premotor cortex. Brain 119:593-609.

Gauthier I, Tarr MJ, Anderson AW, Skudlarski P, Gore JC (1999) Activation of the middle fusiform "face area" increases with expertise in recognizing novel objects. Nat Neurosci 2:568-573.

Greenfield PM (1991) Language, tools, and brain: the ontogeny and phylogeny of hierarchically organized sequential behavior. Behav Brain Sci 14:531-551.

Grezes J, Costes N, Decety J (1999) The effects of learning and intention on the neural network involved in the perception of meaningless actions. Brain 122:1875-1887.

Grill-Spector K, Kourtzi Z, Kanwisher N (2001) The lateral occipital complex and its role in object recognition. Vision Res 41:1409-1422.

Grossman ED, Blake R (2002) Brain areas active during visual perception of biological motion. Neuron 35:1167-1175.

Hanakawa T, Immisch I, Toma K, Dimyan MA, Van Gelderen P, Hallett M
(2003) Functional properties of brain areas associated with motor execution and imagery. J Neurophysiol 89:989-1002.

Hari R, Forss N, Avikainen S, Kirveskari E, Salenius S, Rizzolatti G (1998) Activation of human primary motor cortex during action observation: a neuromagnetic study. Proc Natl Acad Sci USA 95:15061-15065.

Holmes AP, Friston KJ (1998) Generalisability, random effects and population inference. NeuroImage 7:S754.

Hommel B, Müsseler J, Aschersleben G, Prinz W (2002) The theory of event coding (TEC): a framework for perception and action planning. Behav Brain Sci 24:849-878.

Jeannerod M (2001) Neural simulation of action: a unifying mechanism for motor cognition. NeuroImage 14:103-109.

Jeannerod M, Decety J (1995) Mental motor imagery: a window into the representational stages of action. Curr Opin Neurobiol 5:727-732.

Jeannerod M, Arbib MA, Rizzolatti G, Sakata H (1995) Grasping objects: the cortical mechanisms of visuomotor transformation. Trends Neurosci 18:314-320.

Jellema T, Baker CI, Oram MW, Perrett DI (2002) Cell populations in the banks of the superior temporal sulcus of the macaque and imitation. In: The imitative mind (Prinz W, Meltzoff A, eds), pp 267-290. Cambridge, UK: Cambridge UP.

Johnson-Frey SH, Maloof FR, Newman-Norlund R, Farrer C, Inati S, Grafton ST (2003) Actions or hand-object interactions? Human inferior frontal cortex and action observation. Neuron 39:1053-1058.

Jordan MI (1996) Computational aspects of motor control and motor learning. In: Handbook of perception and action: motor skills (Heuer H, Keele S, eds), pp 71-118. New York: Academic.

Josephs O, Turner R, Friston K (1997) Event-related fMRI. Hum Brain Mapp 5:243-248.

Kanwisher N, McDermott J, Chun MM (1997) The fusiform face area: a module in human extrastriate cortex specialized for face perception. J Neurosci 17:4302-4311.

Liberman AM, Cooper FS, Shankweiler DP, Studdert-Kennedy M (1967) Perception of the speech code. Psychol Rev 74:431-461.

Lohmann G, Mueller K, Bosch V, Mentzel H, Hessler S, Chen L, Zysset S, von Cramon DY (2001) Lipsia—a new software system for the evaluation of functional magnetic resonance images of the human brain. Comp Med Imag Graph 25:449-457.

Manthey S, Schubotz RI, von Cramon DY (2003) Premotor cortex in observing erroneous action: an fMRI study. Cognit Brain Res 15:296-307.

Miall RC (2003) Connecting mirror neurons and forward models. NeuroReport 14:2135-2137.

Murata A, Fadiga L, Fogassi L, Gallese V, Raos V, Rizzolatti G (1997) Object representation in the ventral premotor cortex (area F5) of the monkey. J Neurophysiol 78:2226-2230.

Patuzzo S, Fiaschi A, Manganotti P (2003) Modulation of motor cortex excitability in the left hemisphere during action observation: a single- and paired-pulse transcranial magnetic stimulation study of self- and nonself-action observation. Neuropsychologia 41:1272-1278.

Rizzolatti G, Fadiga L (1998) Grasping objects and grasping action meanings: the dual role of monkey rostroventral premotor cortex (area F5). Novartis Found Symp 218:81-103.

Rizzolatti G, Gentilucci M (1988) Motor and visual-motor functions of the premotor cortex. In: Neurobiology of neocortex (Rakic P, Singer W, eds), pp 269-284. Chichester, UK: Wiley.

Rizzolatti G, Riggio L, Dascola I, Umilta C (1987) Reorienting attention across the horizontal and vertical meridians: evidence in favor of a premotor theory of attention. Neuropsychologia 25:31-46.

Rizzolatti G, Camarda R, Fogassi L, Gentilucci M, Luppino G, Matelli M (1988) Functional organization of inferior area 5 in the macaque monkey. II: Area F5 and the control of distal movements. Exp Brain Res 71:491-507.

Rizzolatti G, Fadiga L, Gallese V, Fogassi L (1996) Premotor cortex and the recognition of motor actions. Cognit Brain Res 3:131-141.

Rizzolatti G, Fogassi L, Gallese V (2001) Neurophysiological mechanisms underlying the understanding and imitation of action. Nat Rev Neurosci 2:661-670.

Ruby P, Decety J (2001) Effect of subjective perspective taking during simulation of action: a PET investigation of agency. Nat Neurosci 4:546-550.

Schnitzler A, Salenius S, Salmelin R, Jousmaki V, Hari R (1997) Involvement of primary motor cortex in motor imagery: a neuromagnetic study. NeuroImage 6:201-208. 
Schubotz R (1999) Instruction differentiates the processing of temporal and spatial sequential patterns: evidence from slow wave activity in humans. Neurosci Lett 265:1-4.

Schubotz RI, von Cramon DY (2001) Functional organization of the lateral premotor cortex: fMRI reveals different regions activated by anticipation of object properties, location and speed. Cognit Brain Res 11:97-112.

Schubotz RI, von Cramon DY (2002a) Dynamic patterns make the premotor cortex interested in objects: influence of stimulus and task revealed by fMRI. Cognit Brain Res 14:357-369.

Schubotz RI, von Cramon DY (2002b) Predicting perceptual events activates corresponding motor schemes in lateral premotor cortex: an fMRI study. NeuroImage 15:787-796.

Schubotz RI, von Cramon DY (2002c) A blueprint for target motion: fMRI reveals perceptual complexity to modulate a premotor-parietal network. NeuroImage 16:920-935.

Schubotz RI, von Cramon DY (2003) Functional-anatomical concepts of human premotor cortex: evidence from fMRI and PET studies. NeuroImage 20 [Suppl 1]:120-131.

Schubotz RI, von Cramon DY (2004) Brains have emulators with brains: emulation economized. Behav Brain Sci, in press.

Schubotz RI, von Cramon DY, Lohmann G (2003) Auditory what, where, and when: a sensory somatotopy in lateral premotor cortex. NeuroImage 20:173-185.

Senkfor AJ, Van Petten C, Kutas M (2002) Episodic action memory for real objects: an ERP investigation with perform, watch, and imagine action encoding tasks versus a non-action encoding task. J Cognit Neurosci 14:402-419.

Shima K, Tanji J (2000) Neuronal activity in the supplementary and presupplementary motor areas for temporal organization of multiple movements. J Neurophysiol 84:2148-2160.

Talairach J, Tournoux P (1988) Co-planar stereotaxic atlas of the human brain. New York: Thieme.

Thirion JP (1998) Image matching as a diffusion process: an analogy with Maxwell's demons. Med Image Anal 2:243-260.

Tootell RB, Reppas JB, Kwong KK, Malach R, Born RT, Brady TJ, Rosen BR, Belliveau JW (1995) Functional analysis of human MT and related visual cortical areas using magnetic resonance imaging. J Neurosci 15:3215-3230.

Umiltà MA, Kohler E, Gallese V, Fogassi L, Fadiga L, Keysers C, Rizzolatti G (2001) I know what you are doing: a neurophysiological study. Neuron 31:155-165.

Wohlschläger A, Gattis M, Bekkering H (2003) Action generation and action perception in imitation: an instance of the ideomotor principle. Philos Trans R Soc Lond B Biol Sci 358:501-515.

Worsley KJ, Friston KJ (1995) Analysis of fMRI time-series revisitedagain. NeuroImage 2:173-181.

Yoo SS, Freeman DK, McCarthy JJ 3rd, Jolesz FA (2003) Neural substrates of tactile imagery: a functional MRI study. NeuroReport 14:581-585.

Zysset S, Huber O, Ferstl E, von Cramon DY (2002) The anterior frontomedian cortex and evaluative judgment: an fMRI study. NeuroImage 15: 983-991. 